\title{
Analgesia and Sedation in Critically Ill Adult Patients Admitted to a COVID-19 Intensive Care Unit
}

\author{
Raja Jayaram ${ }^{1}$ \\ ${ }^{1}$ Department of Adult Intensive Care Medicine and Anaesthetics, \\ Oxford University Hospitals NHS Foundation Trust, Oxford, \\ United Kingdom
}

\begin{abstract}
Address for correspondence Raja Jayaram, MD, DPhil, (PhD) Department of Adult Intensive Care Medicine and Anaesthetics, Oxford University Hospitals NHS Foundation Trust, Oxford, United Kingdom (e-mail: raja.jayaram@ndcn.ox.ac.uk).
\end{abstract}

\begin{abstract}
The severe acute respiratory syndrome coronavirus 2 (SARS-CoV-2), causing the COVID-19 has spread globally, prompting world health organization (WHO) to declare COVID-19 a pandemic. As of January 2, 2021, about 82,579,768 laboratory-confirmed COVID-19 cases had been reported to the WHO with 1,818,849 deaths (https:/ covid19.who.int). The pandemic has severely impacted health care systems around the world, resulting in a vast number of surgical procedures being cancelled or postponed and an unprecedented burden on intensive care units (ICU). A critical component of the perioperative or ICU services delivery is the provision of analgesia and sedation.

Keywords

- analgesia and sedation

- COVID-19 intensive care unit

- critically ill adult patients Volatile inhalational anesthetics combined with opioids are widely used in an operating room, whereas in ICUs, intravenous drugs are used for this purpose. Although target-controlled infusions are not routinely used in ICUs, in the context of the thematic series on total intravenous anesthesia during COVID-19 pandemic, this article will focus on key aspects of intravenous sedation and analgesia in the management of critically ill patients admitted to an ICU following positive swab test for SARS-CoV-2 RNA.
\end{abstract}

\section{Introduction}

The severe acute respiratory syndrome coronavirus 2 (SARS-CoV-2), causing the COVID-19 has spread globally, prompting world health organization (WHO) to declare COVID-19 a pandemic. ${ }^{1}$ As of January 2, 2021, about 82,579,768 laboratory-confirmed COVID-19 cases had been reported to the WHO with 1,818,849 deaths (https:// covid19.who.int). The pandemic has severely impacted health care systems around the world, resulting in a vast number of surgical procedures being cancelled or postponed ${ }^{2}$ and an unprecedented burden on intensive care units (ICU). ${ }^{3}$

A critical component of the perioperative or ICU services delivery is the provision of analgesia and sedation. Volatile inhalational anesthetics combined with opioids are widely

published online March 18, 2021
DOI https://doi.org/ 10.1055/s-0041-1726172 ISSN 2457-0206. used in an operating room, ${ }^{4}$ whereas in ICUs, intravenous drugs are used for this purpose. ${ }^{5}$ Although target-controlled infusions (TCI) are not routinely used in ICUs, in the context of the thematic series on total intravenous anesthesia (TIVA) during COIVD-19 pandemic, this article will focus on key aspects of intravenous sedation and analgesia in the management of critically ill patients admitted to an ICU following positive swab test for SARS-CoV-2 RNA.

\section{Pain, Agitation, and Delirium in Intensive Care Unit}

Pain, agitation, and delirium are common in critically ill patients. ${ }^{5,6}$ Contributory factors include the impact of critical illness, ICU procedures such as intubation of the trachea, (c) 2021. Official Publication of The Simulation Society (TSS), accredited by International Society of Cardiovascular Ultrasound (ISCU).

This is an open access article published by Thieme under the terms of the Creative Commons Attribution-NonDerivative-NonCommercial-License, permitting copying and reproduction so long as the original work is given appropriate credit. Contents may not be used for commercial purposes, or adapted, remixed, transformed or built upon. (https://creativecommons.org/licenses/by-nc-nd/4.0/).

Thieme Medical and Scientific Publishers Pvt. Ltd. A-12, 2nd Floor, Sector 2, Noida-201301 UP, India 
mechanical ventilatory support, the establishment of vascular access and routine patient care that necessitates frequent suctioning of the trachea, and change of positioning or physiotherapy. ${ }^{5}$ Patients discharged from an ICU have unfortunately pain as the most common memory linked to their ICU stay period. ${ }^{7}$ On the other hand, agitation and delirium lead to adverse events, including accidental extubation or removal of central and arterial catheters. ${ }^{5}$ Hence, the administration of sedatives and analgesics are integral to the practice of modern intensive care medicine.

The origin of the concept of "intensive care/unit" to manage critically ill with multiorgan failure can be traced to the polio epidemic's impact in the 1950s. ${ }^{8}$ In Blegdam Hospital in Copenhagen, Denmark, an epicenter of the epidemic, patients with respiratory failure were managed by an "iron lung" under deep sedation. ${ }^{9}$ Advancements in ventilator design and technology and development of shorter-acting analgesics, sedatives, and microprocessors, allowing precision in infusions improved respiratory failure management in the following decades. Equally, the adverse clinical outcomes associated with agitation and pain or delirium ${ }^{5,6}$ and morbidity or mortality associated with oversedation ${ }^{10}$ and mechanical ventilation ${ }^{11}$ became increasingly apparent. Moreover, the pharmacokinetics (PK) parameters of many drugs are altered due to the pathophysiological changes associated with critical illness posing challenges when titrating infusions of sedatives and analgesics to maintain optimal sedation and analgesia. ${ }^{12}$

To address these critical issues, a panel of experts, stakeholders, and patient group convened by the Society of Critical Care Medicine, United States published guidelines in the assessment, prevention, and treatment of pain, agitation/sedation, delirium, immobility (mobilization, rehabilitation), and sleep (disruption) in critically ill adults (PADIS guidelines 2018). ${ }^{5}$ Notably, only 2 of the 37 recommendations (addition of neuropathic pain medication to opioids for pain management and avoidance of volatile anesthetics in procedural management) are graded "strong" and inform clinical practice reliably. The rest of conditional recommendations are based on conflicting, or low-quality evidence, applicable to a selected patient subgroup or the potential benefits require balancing against equal risks. Large randomized controlled trials that focus on the choice of sedative agent or level of sedation and impact on mortality in a heterogeneous critically ill population have been published after the 2018 PADIS guidelines, and the results have been neutral. ${ }^{13,14}$

In summary, the choice of sedatives/analgesics, level of sedation, sedation interruption protocols, or objective sedation monitoring tools in ICU remains mostly empirical. Hence, large variations in prescribing patterns among countries and ICUs exist, ${ }^{6,15}$ with the clinical practice determined more by tradition, ingrained methods, unit protocols, and staff familiarity than evidence based. The issue is further compounded by the COVID-19 pandemic with unique patient characteristics; limited understanding of underlying disease mechanisms and pathophysiology; restructuring of organizational and operational models including staffing; requirements for infection control and measures promoting health care workers' safety; and resources limitations.

\section{Analgesia and Sedation in a COVID-19 Intensive Care Unit}

Acute hypoxemic respiratory failure (with or without hypercapnia) necessitating oxygen therapy or mechanical ventilatory support is the most common indication for admission to an ICU following COVID-19. ${ }^{16}$ Notwithstanding the debate whether the underlying COVID-19 pneumonitis is a distinct pathophysiological process ${ }^{17,18}$ or similar to the classical acute respiratory distress syndrome (ARDS), ${ }^{19,20}$ the principles underlying ventilatory and ICU management are same, that is, providing lung-protective mechanical ventilation, prone positioning, use of neuromuscular blockers (NMBs), and consideration for extracorporeal membrane oxygenation (ECMO) in selected patients. ${ }^{21}$

No prospective studies or randomized controlled trials inform the optimum management of sedation and analgesia in patients receiving lung-protective ventilation. Hence, a pragmatic patient-centric approach ensuring comfort, safety, interaction with care providers/family, and promote the ABCDEF bundle 22 is adopted. The approach if not using NMBs would advocate a multimodal regime by using short-acting analgesia (fentanyl preferred given the coexisting risk of acute kidney injury), followed by a sedative infusion (typically propofol or dexmedetomidine and avoiding benzodiazepines) aiming for no to a minimum level of sedation corresponding to a range of +1 to -1 score on Richmond Agitation Sedation Scale ${ }^{23}$ (RASS). If patient ventilator asynchrony despite optimization of settings, increasing agitation in the absence of obvious modifiable/reversible factors or worsening pain (e.g., interval surgical procedure) deeper levels of sedation corresponding to a RASS score -2 to -5 will be needed. Addition of a third drug (midazolam, lorazepam, clonidine, ketamine, and antipsychotics such as haloperidol or quetiapine) may be required to achieve the objective. The choice of the agent, route, intermittent bolus or continuous infusion, and dosage will be guided by the availability, clinical trajectory, cost, familiarity of medical and nursing ICU staff, ICU protocol, patients' age, coexisting organ failures, and comorbidities.

On the other hand, if the administration of NMBs is required (the evidence base for NMBs in ARDS is low quality $^{24}$ ) for optimizing ventilatory management and as a rescue strategy following refractory hypoxemia, best practice advocates establishment of deep levels of sedation, amnesia, and adequate analgesia. Similar considerations apply in patients receiving mechanical lung-protective ventilation in the prone position or on ECMO. However, the use of NMBs has not explicitly been evaluated in prospective studies in these clinical settings. That said, it will be prudent to maintain deep sedation, effective analgesia and adequate muscle relaxation to ensure safety, minimize patient-ventilator asynchrony, and the potential for injuries associated with the change in the position of the head and upper limbs at regular 
intervals in the prone position. Before the commencement of $\mathrm{NMB}$, adequacy of the depth of sedation and analgesia should be assessed by validated tools such as RASS, Behavioral Pain $S_{\text {Scale }}{ }^{25}$, or Critical Care Pain Observation Tool ${ }^{25}$ and achieved levels maintained as long as NMBs are continued. An additional point with practice implication in patients on ECMO will be the alteration in volume of distribution, PK, and bioavailability of drugs, ${ }^{26}$ and hence the need for closer monitoring of sedation levels.

Regardless of the clinical scenario, daily interruption of both sedative and NMB infusions (NMBs first) should be considered. If able to wean, moving toward lighter sedation levels (RASS score $=-1$ to +1 ) should be the priority goal. Unfortunately, these goals have been difficult to achieve during the early phase of the pandemic. Also, the increased use of sedatives, impact of the pandemic on manufacturing, supply chain, or stockpiling by institutions can lead to drug shortages. ${ }^{27}$ Many institutions adopted a nontraditional approach when faced with the challenges by substituting a different agent like benzodiazepines or intermittent boluses of NMBs guided by NMB monitoring. The shift may result in nursing staff, and doctors being unfamiliar with alternative pharmacotherapies, in turn, leading to dosing errors, undersedation or oversedation regardless of patient characteristics.

Whether it contributed to a higher incidence of delirium observed in critically ill patients with COVID- $19^{28}$ is unclear.

\section{Conclusion}

The optimal management of sedation and analgesia in critically ill patients admitted to an ICU, following COVID-19 has been challenging due to paucity of evidence-based guidelines, inability to adhere to best practice recommendations, drug shortages, limited understanding of the disease process, unique patient characteristics, staffing issues, organizational restructuring, barriers, and resource constraints. Equally, COVID-19 pandemic demonstrated that the critical care community came together with admirable focus, energy, teamwork, and resilience when faced with a crisis. With remarkable advances made in all key domains of discovery and progress achieved, including vaccine development with immunization campaigns already underway in many countries, there is hope in the horizon. Until then, as Albert Camus wrote in "The Plague," I have no idea what's awaiting me, or what will happen when this all ends. For the moment I know this: there are sick people and they need curing.

\section{Funding \\ None.}

\section{Conflict of Interest}

None declared.

\section{References}

1 Cucinotta D, Vanelli M. WHO declares COVID-19 a pandemic. Acta Biomed 2020;91(1):157-160
2 Collaborative CO; COVIDSurg Collaborative. Elective surgery cancellations due to the COVID-19 pandemic: global predictive modelling to inform surgical recovery plans. $\mathrm{Br}$ J Surg 2020;107(11):1440-1449

3 Tan E, Song J, Deane AM, Plummer MP. Global impact of coronavirus disease 2019 infection requiring admission to the ICU: a systematic review and meta-analysis. Chest 2020;S0012-3692(20)34906-0

4 Miller AL, Theodore D, Widrich J, Inhalational Anesthetic. Treasure Island, FL: StatPearls; 2020

5 Devlin JW, Skrobik Y, Gélinas C, et al. Clinical practice guidelines for the prevention and management of pain, agitation/sedation, delirium, immobility, and sleep disruption in adult patients in the ICU. Crit Care Med 2018;46(9):e825-e873

6 Reade MC, Finfer S. Sedation and delirium in the intensive care unit. N Engl J Med 2014;370(5):444-454

7 Stein-Parbury J, McKinley S. Patients' experiences of being in an intensive care unit: a select literature review. Am J Crit Care 2000;9(1):20-27

8 Weil MH, Tang W. From intensive care to critical care medicine: a historical perspective. Am J Respir Crit Care Med 2011;183(11):1451-1453

9 Ibsen B. The anaesthetist's viewpoint on the treatment of respiratory complications in poliomyelitis during the epidemic in Copenhagen, 1952. Proc R Soc Med 1954;47(1):72-74

10 Shehabi Y, Bellomo R, Kadiman S, et al; Sedation Practice in Intensive Care Evaluation (SPICE) Study Investigators and the Australian and New Zealand Intensive Care Society Clinical Trials Group. Sedation intensity in the first 48 hours of mechanical ventilation and 180-day mortality: a multinational prospective longitudinal cohort study. Crit Care Med 2018;46(6):850-859

11 Haribhai S, Mahboobi SK, Ventilator Complications. Treasure Island, FL: StatPearls; 2020

12 Tse AHW, Ling L, Lee A, Joynt GM. Altered pharmacokinetics in prolonged infusions of sedatives and analgesics among adult critically ill patients: a systematic review. Clin Ther 2018;40(9):1598-1615.e2

13 Shehabi Y, Howe BD, Bellomo R, et al; ANZICS Clinical Trials Group and the SPICE III Investigators. Early sedation with dexmedetomidine in critically ill patients. $\mathrm{N}$ Engl J Med 2019;380(26):2506-2517

14 Olsen HT, Nedergaard HK, Strøm T, et al. Nonsedation or light sedation in critically ill, mechanically ventilated patients. N Engl J Med 2020;382(12):1103-1111

15 Chawla R, Myatra SN, Ramakrishnan N, Todi S, Kansal S, Dash SK. Current practices of mobilization, analgesia, relaxants and sedation in Indian ICUs: a survey conducted by the Indian Society of Critical Care Medicine. Indian J Crit Care Med 2014;18(9):575-584

16 Phua J, Weng L, Ling L, et al; Asian Critical Care Clinical Trials Group. Intensive care management of coronavirus disease 2019 (COVID-19): challenges and recommendations. Lancet Respir Med 2020;8(5):506-517

17 Gattinoni L, Chiumello D, Rossi S. COVID-19 pneumonia: ARDS or not? Crit Care 2020;24(1):154

18 Gattinoni L, Coppola S, Cressoni M, Busana M, Rossi S, Chiumello D. COVID-19 does not lead to a "typical" acute respiratory distress syndrome. Am J Respir Crit Care Med 2020;201(10):1299-1300

19 Ferrando C, Suarez-Sipmann F, Mellado-Artigas R, et al COVID-19 Spanish ICU Network. Clinical features, ventilatory management, and outcome of ARDS caused by COVID-19 are similar to other causes of ARDS. Intensive Care Med 2020;46(12):2200-2211

20 Goligher EC, Ranieri VM, Slutsky AS. Is severe COVID-19 pneumonia a typical or atypical form of ARDS? And does it matter? Intensive Care Med 2020 
21 Fan E, Beitler JR, Brochard L, et al. COVID-19-associated acute respiratory distress syndrome: is a different approach to management warranted? Lancet Respir Med 2020;8(8):816-821

22 Pandharipande P, Banerjee A, McGrane S, Ely EW. Liberation and animation for ventilated ICU patients: the ABCDE bundle for the back-end of critical care. Crit Care 2010;14(3):157

23 Sessler CN, Gosnell MS, Grap MJ, et al. The Richmond Agitation-Sedation Scale: validity and reliability in adult intensive care unit patients. Am J Respir Crit Care Med 2002;166(10):1338-1344

24 Alhazzani W, Belley-Cote E, Møller MH, et al. Neuromuscular blockade in patients with ARDS: a rapid practice guideline. Intensive Care Med 2020;46(11):1977-1986
25 Gélinas C, Joffe AM, Szumita PM, et al. A psychometric analysis update of behavioral pain assessment tools for noncommunicative, critically ill adults. AACN Adv Crit Care 2019;30(4):365-387

26 Shekar K, Fraser JF, Smith MT, Roberts JA. Pharmacokinetic changes in patients receiving extracorporeal membrane oxygenation. J Crit Care 2012;27(6):741.e9-741.e18

27 Adams CD, Altshuler J, Barlow BL, et al. Analgesia and sedation strategies in mechanically ventilated adults with COVID-19. Pharmacotherapy 2020;40(12):1180-1191

28 Helms J, Kremer S, Merdji H, et al. Delirium and encephalopathy in severe COVID-19: a cohort analysis of ICU patients. Crit Care 2020;24(1):491 